\title{
Atomistic simulation of the nano-structural evolution of Raney-type catalysts from spray-atomized NiAl precursor alloys during leaching with $\mathrm{NaOH}$ solution
}

\author{
N. C. Barnard ${ }^{1}$, S. G. R. Brown ${ }^{1}$, F. Devred ${ }^{2}$, B. E. Nieuwenhuys ${ }^{2}$ \\ \& J. W. Bakker ${ }^{2}$ \\ ${ }^{1}$ Materials Research Centre, School of Engineering, Swansea University, \\ UK \\ ${ }^{2}$ Leiden Institute of Chemistry, Leiden University, The Netherlands
}

\begin{abstract}
A model for the nano-structural evolution of Raney-type nickel catalysts (widely used in hydrogenation reactions) from the constituent intermetallic phases present in nickel-aluminium precursor alloys is presented here. Nano-porous nickel catalysts are prepared via a caustic leaching process where the $\mathrm{NiAl}$ alloy powder (typically 50-50 at.\%) is immersed in concentrated $\mathrm{NaOH}$ solution in order to leach away the aluminium present to leave a highly-porous nickel catalyst (often referred to as spongy nickel).

The temporal evolution of the nickel catalyst is described by a kinetic Monte Carlo (kMC) method, which captures two dominant processes taking place on leaching with $\mathrm{NaOH}$ solution: rapid aluminium dissolution from the crystalline intermetallic phases and surface adatom diffusion of nickel and aluminium. Structural changes from $\mathrm{NiAl}_{3}$ and $\mathrm{Ni}_{2} \mathrm{Al}_{3}$ phases present in the starting alloy are considered on an event-by-event basis. Individual crystallite size, pore diameter and effective surface area are predicted. Finally, a Metropolis Monte Carlo method is used to investigate the surface nature of the as-leached catalyst and compared to concurrent experimental investigations.
\end{abstract}

Keywords: de-alloying, leaching, Raney-Ni catalysts, Monte Carlo simulation. 


\section{Introduction}

Raney-type nickel catalysts are typically prepared by leaching aluminium from a $\mathrm{Ni}-\mathrm{Al}$ alloy using a concentrated sodium hydroxide solution [1-3]. This process of activation critically affects the structure and properties of Raney-type nickel catalysts. The initial structure and composition of the starting alloy also influence the performance of the final catalyst [4-7]. In this paper, numerical modelling is compared to experimental measurements in an attempt to simulate both the 3D morphology of as-leached Raney-Ni catalyst material and investigate the nature of the exposed catalyst surfaces.

Firstly, a kinetic Monte Carlo (kMC) [8] for the nano-structural evolution of so-called spongy nickel from the constituent intermetallic phases present in nickel-aluminium precursor alloys is described. Experimental data concerning nano-porous nickel catalyst powder used in this paper are derived from leached $\mathrm{NiAl}$ alloy powder produced via a spray-atomization route rather than the conventional cast-and-crushed route.

The temporal evolution of the nickel catalyst during de-alloying described by the $\mathrm{kMC}$ model captures two dominant processes taking place during leaching with $\mathrm{NaOH}$ solution (i) de-alloying via rapid aluminium dissolution from the crystalline intermetallic phases and (ii) surface adatom diffusion of nickel and aluminium. Structural changes from $\mathrm{NiAl}_{3}$ and $\mathrm{Ni}_{2} \mathrm{Al}_{3}$ phases present in the starting alloy are considered on an event-by-event basis. Individual crystallite size, pore diameter and effective surface area are predicted and compared to concurrent experimental investigations [9].

In its role as a catalyst, the surface condition of the as-leached nickel nanostructure is highly important. In particular, the sites occupied on the surface of the catalyst by any residual aluminium atoms are of interest. In order to investigate the surface configuration of as-leached surfaces a Metropolis Monte Carlo (MMC) model is subsequently applied. As a starting point the MMC model exploits the nano-porous structure already predicted by the kMC approach. The MMC model aims to simulate the appearance of Al-rich surface configurations that are routinely observed in experiment.

\section{Numerical models}

\subsection{Kinetic Monte Carlo (kMC) method}

The Monte Carlo model is based on a technique originally used to model the leaching of silver from a $\mathrm{Au}-50$ at.\% Ag alloy, examining the evolution of nano-porosity [10]. For NiAl alloys two key processes are assumed to be taking place during leaching, namely adatom diffusion ( $\mathrm{Ni}$ or $\mathrm{Al}$ ) and $\mathrm{Al}$ dissolution. These processes are assumed to occur at known rates that serve as the inputs for the model. Both diffusion and dissolution of adatoms are assumed to proceed at a rate described by the following Arrhenius relationships respectively:

$$
k_{n}^{\text {diff }}=v_{\text {diff }} \exp \left(-\frac{n E_{b}}{k_{b} T}\right)
$$




$$
k_{n}^{\text {diss }}=v_{\text {diss }} \exp \left(-\frac{n E_{b}}{k_{b} T}\right)
$$

where $k_{n}^{\text {diff }}$ is the rate constant for diffusion of an adatom ( $\mathrm{Ni}$ or $\left.\mathrm{Al}\right), k_{n}^{\text {diss }}$ is the rate constant for dissolution of an adatom (Al only), $v_{\text {diff }}$ and $v_{\text {diss }}$ are frequency factors $\left(1 \times 10^{13} \mathrm{~s}^{-1}\right.$ and $1 \times 10^{17} \mathrm{~s}^{-1}$ respectively $), n$ is the number of bonds, $k_{b}$ is the Boltzmann constant and $T$ is absolute temperature $(\mathrm{K})$. The value of $1 \times 10^{17} \mathrm{~s}^{-1}$ is at the higher end of reported frequency factors [11]. The bond energies are taken as $E_{b}=0.165,0.1875$ and $0.069 \mathrm{eV}$ for $\mathrm{Ni}-\mathrm{Ni}, \mathrm{Ni}-\mathrm{Al}$ and $\mathrm{Al}-\mathrm{Al}$ bonds respectively. These values are based on published pair potentials [12] and calculated over the number of nearest neighbour atoms [13]. In the present model it is assumed that the crystal is without any type of lattice defect and the role of any reaction products is not included. Furthermore, it should be noted that the model does not include recently proposed fragmentation mechanisms that may occur during leaching [14].

The surface diffusion of both elements during leaching means that the final nano-metric spongy nickel structure is not 'revealed' but 'constructed' during leaching by diffusion of $\mathrm{Ni}$ adatoms. Equations 1 and 2 ensure that atoms with fewer bonds are more likely to dissolve or diffuse. Adatoms either already present in, or diffusing to, lower energy sites (i.e. sites of higher coordination number) will tend to become less likely to diffuse further. In this way $\mathrm{Ni}$ adatoms tend to cluster together at surfaces that are increasingly exposed during Al dissolution, thus building up a nano-scopic structure. Knowledge of the rates of all permitted transitions allows the time increment for any iteration of the model to be calculated. This has been done using the well-known Bortz-KalosLiebowitz (BKL) algorithm described before [8].

To carry out a simulation a 3D computational domain is created containing a total of $200^{3} \mathrm{Ni}$ and $\mathrm{Al}$ atoms. Initially a cubic region of material is created, all faces of which are considered to be exposed to concentrated $\mathrm{NaOH}$ solution. There are three phases usually encountered in the precursor NiAl alloy powders: (i) $\mathrm{NiAl}_{3}$ phase, which is represented by an $\mathrm{L}_{2}$ structure with an $A B C A B C$ stacking sequence, (ii) $\mathrm{Ni}_{2} \mathrm{Al}_{3}$ phase, the structure of which is a five-layer closepacked unit cell with stacking $A B C B C A$, (iii) Al-rich eutectic phase which is assumed to leach away very quickly and is not dealt with in the model. kMC simulations of the $\mathrm{NiAl}_{3}$ and $\mathrm{Ni}_{2} \mathrm{Al}_{3}$ phases are used here.

Once the BKL algorithm is applied $\mathrm{Al}$ atoms are progressively removed (at a rate that decreases with time) (8). Simultaneously, adatom diffusion of Ni results leads to the development of a nano-porous structure.

Figure 1 shows the final predicted as-leached structure of the $\mathrm{NiAl}_{3}$ precursor material after 3 hours of leaching at $353 \mathrm{~K}$ showing the nano-porous structure that has developed. Figure 2 shows the results of a simulation for $\mathrm{Ni}_{2} \mathrm{Al}_{3}$ precursor material leached under the same conditions, a nano-porous structure has developed but is much less open than the structure from the $\mathrm{NiAl}_{3}$ precursor material. These two predicted morphologies are used as the starting point for MMC calculations. In both figures the nano-porous structure has surfaces which are almost exclusively exposed (100) or exposed (111) planes (coordination 
numbers of 8 and 9 respectively). Atoms with coordination numbers of 7 are also common along the ridges at the edges of these planes.

\subsection{Metropolis Monte Carlo (MMC) method}

In order to address the experimental observations that leached Raney-Ni catalytic powders containing $<10 \% \mathrm{Al}$, can nevertheless possess $30-50 \%$ metallic $\mathrm{Al}$ at the surface a Metropolis Monte Carlo modelling approach has been adopted. The MMC algorithm differs from the kinetic Monte Carlo method in that it attempts to determine the minimum energy for a system, but without the specific reference to time. The $\mathrm{kMC}$ technique has been used to simulate the time evolution of nano-porous structures during leaching. In contrast, the MMC technique is used here to determine the lowest energy configuration for these computed structures in terms of optimum interatomic spacings and distribution of atomic species.

The general MMC algorithm is described widely in the literature, e.g. [15]. Here it is used in canonical ensemble mode. The number of atoms, N, temperature $(300 \mathrm{~K})$ and pressure are fixed. The number of each kind of atom $(\mathrm{Ni}$ or Al) is also fixed. Two types of trial are performed.

(1) Random displacement of each atom in the computational domain from its current position. The magnitude of this displacement is of the order $0.003 \AA$. Once each atom has been displaced the decision on acceptance of the new configuration is based on the standard Metropolis method:

$$
\frac{P_{\text {new }}}{P_{\text {old }}}=\exp \left(\frac{-\Delta U}{k T}\right)
$$

Where $k T$ is the Boltzmann factor and $\Delta U$ is the potential energy difference. If $P_{\text {new }} / P_{\text {old }}>1$ the new configuration is accepted. If not it is still accepted with a probability of $P_{\text {new }} / P_{\text {old }}$.

(2) An atom is exchanged with another atom selected at random. The decision on acceptance of the change is also according to equation 3 above.

After all atoms in the simulation have undergone several of these steps the lattice parameter of one box direction, [001] [010] or [100], is selected at random and altered. Random changes in lattice parameter of up to $1 \AA$ are used. The relative probability of acceptance of such an alteration is given by:

$$
\frac{P_{\text {new }}}{P_{\text {old }}}=\exp \left(\frac{-(\Delta U+P \Delta V-N k T \ln V)}{k T}\right)
$$

Here $\mathrm{V}$ is the volume of the box and $\mathrm{P}$ is pressure (assumed to be zero). These trials are repeated until the system effectively reaches steady state. While several data sets have been made available to model NiAl alloys we choose the tightbinding (TB) second-moment approximation (SMA) approach adopted by Papanicolaou et al. [16]. This TB-SMA approach defines the total energy of the system ( $U$ in equation 3 ) as

$$
U=-\sum_{i=1}^{N}\left(\sum_{j \neq i} A_{\alpha \beta} \exp \left[-p_{\alpha \beta}\left(\frac{r_{i j}}{r_{0}^{\alpha \beta}}-1\right)\right]-\left\{\sum_{j \neq i} \xi_{\alpha \beta}^{2} \exp \left[-2 q_{\alpha \beta}\left(\frac{r_{i j}}{r_{0}^{\alpha \beta}}-1\right)\right]\right\}^{1 / 2}\right)
$$


Here the first term is a pair-potential repulsive term and the second term corresponds to the band-structure term. $\mathrm{N}$ is the total number of atoms, $\mathrm{r}_{\mathrm{ij}}$ is the distance between atoms $i$ and $j$ of the species $\alpha$ and $\beta$, respectively, ( $\alpha$ and $\beta$ stand for $\mathrm{Al}$ and $\mathrm{Ni}$ ) and the sum $\mathrm{j}$ is extended up to fifth neighbours. Numerical values of the constants $\mathrm{A}, \xi$, p, $\mathrm{q}$ and $\mathrm{r}_{0}$ are given in [16]. MMC simulations of this type have been carried out in the past to investigate the surface configurations of isolated nano-clusters of $\mathrm{Ni}_{\mathrm{x}} \mathrm{Al}_{\mathrm{y}}$ alloys [17].

In summary, a kMC model is used to generate a nano-porous structure, hopefully representative of real as-leached spongy nickel. The predicted 3D arrangement is then used as a reference structure on which different proportions of $\mathrm{Ni}$ and $\mathrm{Al}$ atoms can be placed. While maintaining the numbers of $\mathrm{Al}$ and $\mathrm{Ni}$ atoms constant, the MMC algorithm repeatedly swaps $\mathrm{Al}$ and $\mathrm{Ni}$ atoms whilst also adjusting the overall lattice parameters to compute the lowest energy configuration for the given atomic arrangement and relative proportions of $\mathrm{Al}$ and $\mathrm{Ni}$ atoms. No further diffusion or leaching occurs during the MMC simulation. The MMC model is thus used in a similar manner to published work on isolated nano-clusters of different compositions [17]. The difference here is that the MMC simulation will be used on the non-spheroidal clusters predicted from $\mathrm{kMC}$.

\section{Results and discussion}

In order to compare simulation results to experimental measurements data related to as-leached catalyst morphology as well as data related to surface composition is required. For morphological comparison a TEM bright field image is used. Prior to the structural characterization the leached samples were slowly passivated with oxygen. The image was taken with a Philips CM 20, equipped with a LaB6 cathode and operated at $200 \mathrm{kV}$ accelerating voltage. This information is combined with Brunauer, Emmett and Teller method (BET) measurements providing surface area data. For surface composition information X-ray photoelectron spectroscopy (XPS) data is used. Figure 3 shows a TEM image of an as-leached particle where two distinct regions can be seen. To the right is a relatively open structure that has formed from $\mathrm{NiAl}_{3}$ phase. To the left is a denser region formed from $\mathrm{Ni}_{2} \mathrm{Al}_{3}$ phase. In Figure 3 the box region to the left of $\mathrm{A}$ is the computed $\mathrm{kMC}$ nano-porous structure from figure 2 overlaid onto the TEM image at the corresponding magnification. The box region to the left of $\mathrm{B}$ is the computed $\mathrm{kMC}$ nano-porous structure from figure 1 again at the corresponding magnification. A visual comparison indicates that the predicted nano-porous structures are similar to those observed in a real particle.

Before considering surface area a note of warning must be sounded. In precursor alloys containing $\mathrm{Ni}_{2} \mathrm{Al}_{3}$ phase it has been reported that the $\mathrm{Ni}_{2} \mathrm{Al}_{3}$ phase does not leach properly and is still present in the final catalyst [18-20]. This would therefore confuse interpretation of results. Consequently, in this paper we compare to BET measurements made on catalyst powder made from a $\mathrm{Ni}-\mathrm{Al} 82.5$ at.\% precursor material (with a particle size in the range 106-150 $\mu \mathrm{m})$. This alloy does not contain any $\mathrm{Ni}_{2} \mathrm{Al}_{3}$ phase and thus can safely be 
assumed to have properly leached. Table 1 shows both bulk and surface compositions for this material as well as the surface area per weight from BET. The structures predicted from the kMC model (Figures 1 and 2) have estimated surface areas of 49.9 and $49.8 \mathrm{~m}^{2} \mathrm{~g}^{-1}$ respectively, showing that despite its simplicity the model is capable of providing results at the correct length scale.

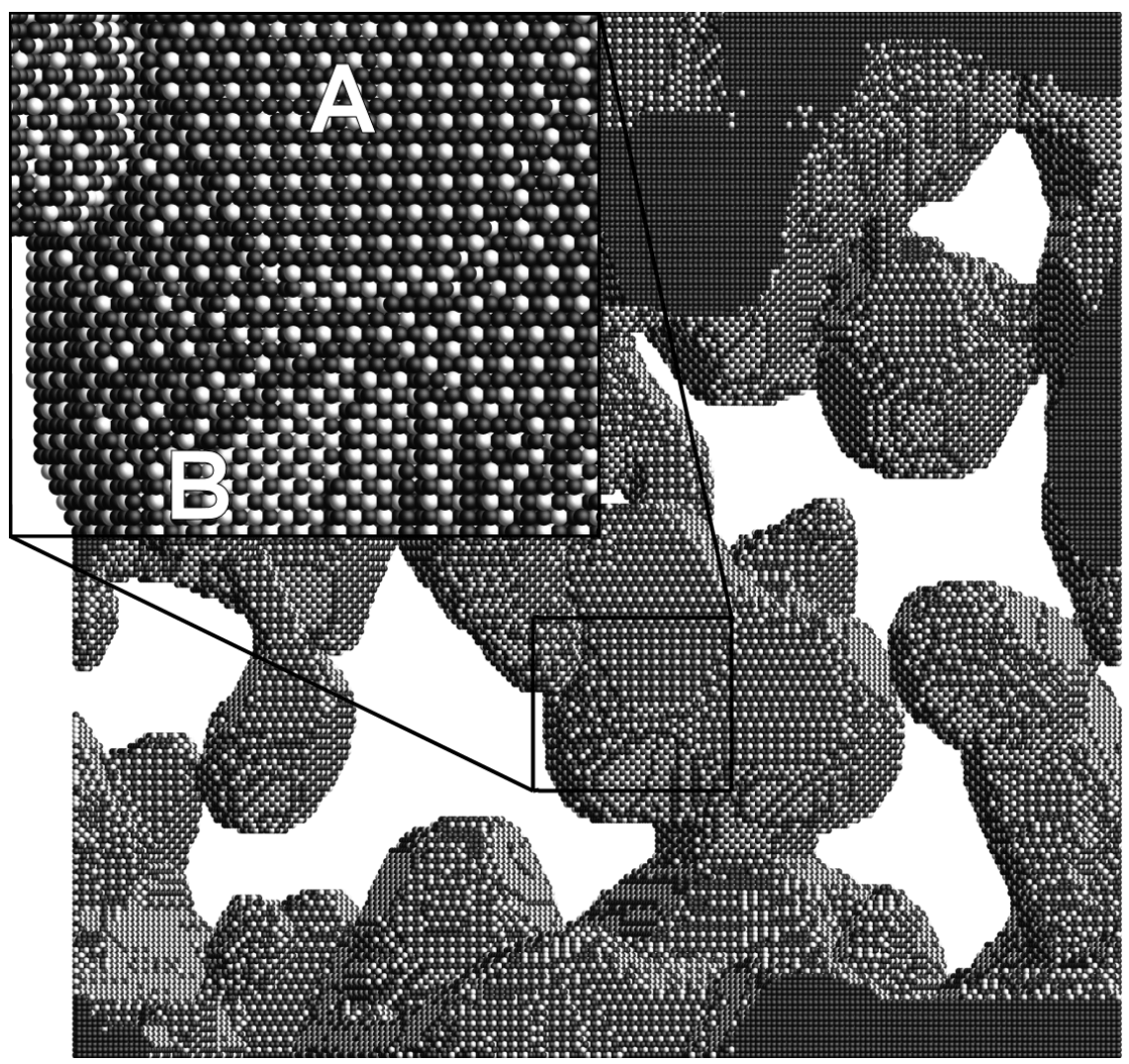

Figure 1: $3 \mathrm{D}$ predicted nano-porous structure of leached $\mathrm{NiAl}_{3}$ precursor material. The box at the top left shows a magnified view. Region $\mathrm{A}$ is an exposed (111) plane and just to the right of $\mathrm{B}$ is an exposed (100) plane. Dark atoms are Ni and light atoms are Al. Edge length of the box is $50 \mathrm{~nm}$.

Table 1: $\quad$ Measured bulk and surface data for an as-leached catalyst powder manufactured from spray-atomized $\mathrm{Ni}-\mathrm{Al} 82.5$ at.\% precursor alloy.

\begin{tabular}{|c|c|c|c|c|c|}
\hline Catalysts & $\begin{array}{c}\text { Ni (at \%) } \\
\text { Bulk }\end{array}$ & $\begin{array}{c}\text { Al (at \%) } \\
\text { Bulk }\end{array}$ & $\begin{array}{c}\text { Ni (at \%) } \\
\text { XPS }\end{array}$ & $\begin{array}{c}\text { Al (at \%) } \\
\text { XPS }\end{array}$ & $\begin{array}{c}\text { BET } \\
\left(\mathrm{m}^{2} \mathrm{~g}^{-1}\right)\end{array}$ \\
\hline $\begin{array}{c}\text { Ni-Al 82.5at.\% } \\
(106-150 \mu \mathrm{m})\end{array}$ & $95 \pm 1$ & $5 \pm 1$ & $68 \pm 1$ & $32 \pm 1$ & $48 \pm 2$ \\
\hline
\end{tabular}


Table 1 shows a very interesting result. Although the powder has a bulk Al content of $5 \pm 1$ at. $\%$ this has risen to $32 \pm 1$ at. $\% \mathrm{Al}$ at the surface. To simulate this effect the MMC model was run on an isolated nano-cluster 54 atoms in diameter (which equates to a sphere with approximately $50 \mathrm{~m}^{2} \mathrm{~g}^{-1}$ ). The nanocluster was set up as an FCC Ni structure with 5 at.\% Al present distributed randomly within the particle. Figure 4 shows the nano-cluster once the MMC model has effectively converged to the lowest energy configuration. The final predicted lattice parameter from the model is $0.343 \mathrm{~nm}$ with a predicted cohesive energy per atom of $4.38 \mathrm{eV}$, in good agreement with previous published results [16]. The final surface composition of the nano-cluster is 29.5 at.\% Al where importantly there are no Al-Al bonds present.

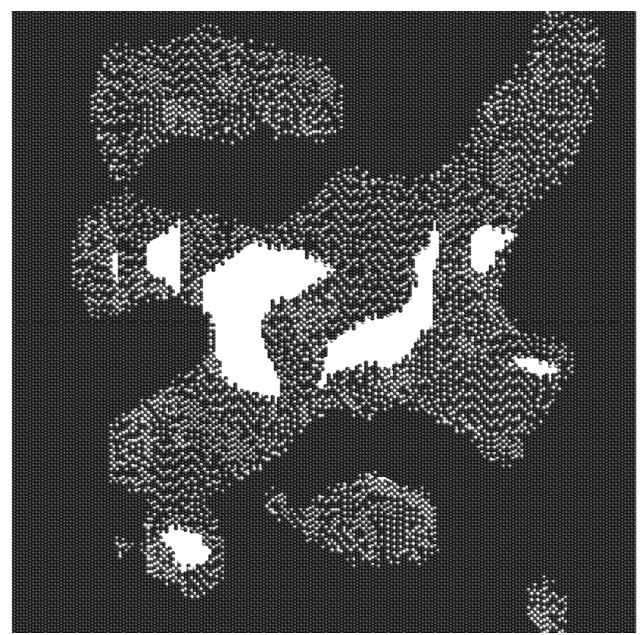

Figure 2: $\quad 3 \mathrm{D}$ predicted nano-porous structure of leached $\mathrm{Ni}_{2} \mathrm{Al}_{3}$ precursor material. Dark atoms are Ni and light atoms are Al. Edge length of the box is $50 \mathrm{~nm}$.

This predicted surface composition is thus in very good agreement with the BET measurement. Interestingly, the nano-cluster actually contains enough Al atoms to provide a 69 at.\% surface coverage of Al. However, this would entail Al-Al bonding which is not energetically favourable [17].

This last result provides the possibility of estimating the surface configuration of predicted leached structures. Segregation of Al to the surface is energetically favourable, provided there are no Al-Al bonds. In Figure 1 we have a structure which is 95 at.\% Ni. As many as possible of the Al atoms have been placed at surface sites ensuring that no Al-Al bonds are present. As a result, the predicted structure in Figure 1 has a surface area per gram of $49.9 \mathrm{~m}^{2} \mathrm{~g}^{-1}$, a bulk $\mathrm{Al}$ composition of 5 at. $\%$ and a surface Al composition of 28.9 at. $\%$ all in very close agreement with the experimental data in Table 1.

As an aside, the measured value [21] of surface aluminium content for an exposed (111) plane is 25 at.\% $\left(\mathrm{Ni}_{90} \mathrm{Al}_{10}\right.$ at $\left.1100 \mathrm{~K}\right)$. A theoretical maximum of 
surface aluminium content for an exposed (100) plane, precluding any Al-Al bonds, is 50 at.\%. Since a structure predominantly comprising exposed (111) and (100) planes is predicted, it might therefore be expected that as-leached Raney$\mathrm{Ni}$ powders with residual $\mathrm{Al}$ would possess surface concentrations of $\mathrm{Al}$ in the range $25-50$ at.\% which is what is routinely observed.

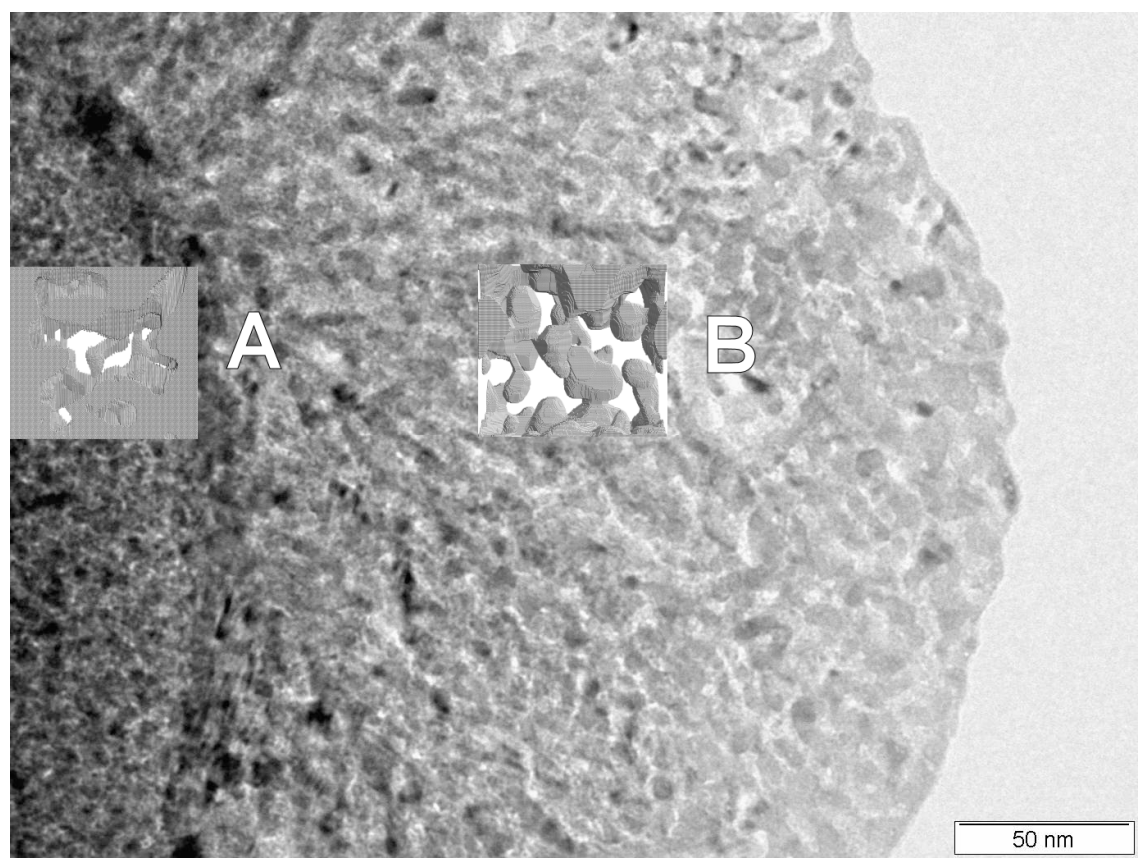

Figure 3: TEM bright field image of leached NiAl alloy where two distinct regions can be seen. To the right is a relatively open structure that has formed from $\mathrm{NiAl}_{3}$ phase. To the left is a denser region formed from $\mathrm{Ni}_{2} \mathrm{Al}_{3}$ phase. (TEM image courtesy of Dr. Ute Hörmann, Ulm University, Germany.)

\section{Conclusions}

- A kinetic Monte Carlo model has been shown to be capable of simulating as-leached nano-porous structures that develop during leaching of NiAl alloys.

- As shown by previous workers the Metropolis Monte Carlo calculations indicate that segregation of any residual $\mathrm{Al}$ to the surface is energetically favourable provided that no Al-Al bonds form.

- Simulated surface area per gram of catalyst and simulated surface concentration of $\mathrm{Al}$ are both in good quantitative agreement with measurements carried out on a leached NiAl precursor alloy that did not contain $\mathrm{Ni}_{2} \mathrm{Al}_{3}$ phase. 


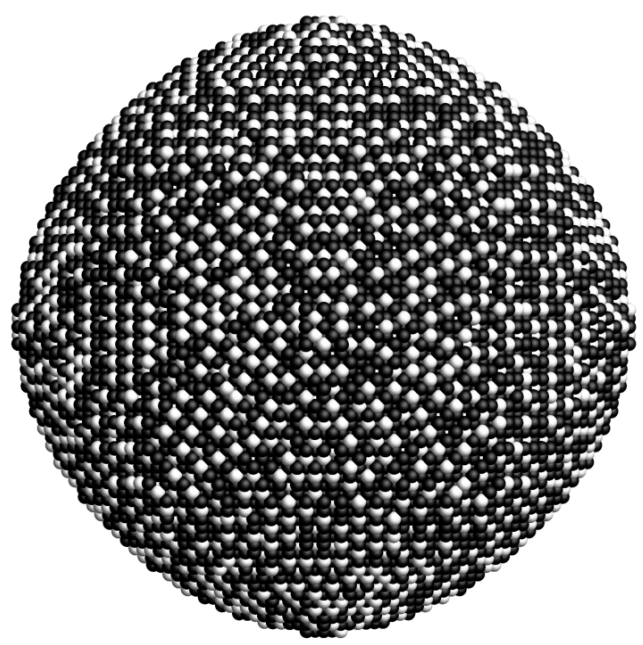

Figure 4: $\quad$ Isolated NiAl nano-cluster 54 atoms in diameter (equating to a sphere approximately $50 \mathrm{~m}^{2} \mathrm{~g}^{-1}$ ) with an FCC structure, bulk composition 5 at.\% Al. Dark atoms are Ni and light atoms are Al. The final surface composition computed via the MMC model is 29.5 at.\% Al. NB there are no Al-Al bonds present.

- $\quad$ The predicted surface condition of leached NiAl material is one with predominantly (111) and (100) exposed planes where residual Al is present with no Al-Al bonds.

\section{Acknowledgements}

The authors would like to thank Dr Ute Hörman of Ulm University in Germany for kindly providing the TEM image. This work has been carried out as part of the Intermetallic Materials Processing in Relation to Earth and Space Solidification (IMPRESS) European integrated project (contract no. NM3-CT2004-500635) coordinated by the European Space Agency (ESA) [22].

\section{References}

[1] M. Raney, US Patent 1.563.787 (1925).

[2] P. Fouilloux, G.A. Martin, A.J. Renouprez, B. Moraweck, B. Imelik and M. Prettre, J. Catal, 25 (1972) 212.

[3] A.J Smith and D.L. Trimm, Annu. Rev. Mater. Res. 35 (2005) 127.

[4] A.B. Fasman, V.F. Timofeeva, V.N. Rechkin and Y.F. Klyuchnikov, Kyn. and Khem. 13 (1971) 1513.

[5] J. Freel, W.J.M. Pieters and R.B. Anderson, J. Catal. 14 (1969) 247.

[6] S. Sane, J.M. Bonnier, J.P. Damon and J. Masson, Appl. Catal. 9 (1984) 69. 
[7] M.S. Wainwright, Handbook of Heterogeneous Catalysis, Vol 1, G. Ertl, H. Knozinger and J. Weitkamp (Eds), VCH Publ., New York (1997), ISBN 3527-29212-8.

[8] N.C. Barnard and S.G.R. Brown, Simulation of Electrochemical Process II, V.G. DeGiorgi, C.A. Brebbia and R.A. Adey (Eds), WIT Press, Southampton, UK (2007), ISBN 978-1-84564-071-2, 53.

[9] F. Devred, A.H. Gieske, N. Adkins, J.W. Bakker, B.E. Nieuwenhuys, Applied Catalysis A, General 356 (2009) 154.

[10] J. Erlebacher, M. J. Aziz, A. Karma, N. Dimitrov and K. Sieradzki, Nature, (2001) 410: 450 .

[11] D. D. MacNeil and J. R. Dahn, J. Phys. Chem., A (2001), 105, 4430.

[12] J. Mei, Bernard R. Cooper and S. P. Lim, Phys. Rev. B, 54, 1, (1996), 178.

[13] M. I. Baskes and C. F. Melius, Phys. Rev. B, 20, 8, (1979), 3197.

[14] R. Wang, H. Chen, Z. Lu, S. Qiu and T. Ko, J. Mat. Sci. 43 (2008) 5712.

[15] M. P. Allen and D. Tildesley, Computer Simulation of Liquids, Clarendon, Oxford, 1987.

[16] N. I. Papanicolaou, H. Chamati, G. A. Evangelakis and D. A. Papaconstantopoulos, Comp. Mat. Sci., 27 (2003) 191.

[17] E. E. Zhurkin and M. Hou, J. Phys. Condens. Matter, 12 (2000) 6735.

[18] H. Lei, Z. Song, D. Tan, X. Bao, X. Mu, B. Zong and E. Min, Appl. Cat. A: General 214 (2001) 69.

[19] M. L. Bakker, D. J. Young and M.S. Wainwright, J. Mat. Sci. 23, (1988) 3921.

[20] R. Wang, Z. Lu and T. Ko, J. Mat. Sci. 36 (2001) 5649.

[21] T. Schulthess and R. Monnier, Phys. Rev. B, 50, 24, (1994), 18564.

[22] D.J. Jarvis and D. Voss, Mat. Sci. Eng. A, 413-414, (2005), 583. 